\title{
Oct4 resetting by Aurkb-PP1 cell cycle axis determines the identity of mouse embryonic stem cells
}

\author{
Jihoon $\operatorname{Shin}^{1}$ \& Hong-Duk Youn ${ }^{1,2, *}$ \\ ${ }^{1}$ National Creative Research Center for Epigenome Reprogramming Network, Department of Biomedical Sciences, Ischemic/Hypoxic \\ Disease Institute, Seoul National University College of Medicine, ${ }^{2}$ Department of Molecular Medicine \& Biopharmaceutical Sciences, \\ Graduate School of Convergence Science, Seoul National University, Seoul 03080, Korea
}

In embryonic stem cells (ESCs), cell cycle regulation is deeply connected to pluripotency. Especially, core transcription factors (CTFs) which are essential to maintaining the pluripotency transcription programs should be reset during $M / G 1$ transition. However, it remains unknown about how CTFs are governed during cell cycle progression. Here, we describe that the regulation of Oct4 by Aurora kinase b (Aurkb)/protein phosphatase 1 (PP1) axis during the cell cycle is important for resetting Oct4 to pluripotency and cell cycle related target genes in determining the identity of ESCs. Aurkb starts to phosphorylate Oct4(S229) at the onset of G2/M phase, inducing the dissociation of Oct4 from chromatin, whereas PP1 binds Oct4 and dephosphorylates Oct4(S229) during M/G1 transition, which resets Oct4-driven transcription for pluripotency and the cell cycle. Furthermore, Aurkb phosphormimetic and PP1 binding-deficient mutations in Oct4 disrupt the pluripotent cell cycle, lead to the loss of pluripotency in ESCs, and decrease the efficiency of somatic cell reprogramming. Based on our findings, we suggest that the cell cycle is directly linked to pluripotency programs in ESCs. [BMB Reports 2016; 49(10): 527-528]

ESCs proliferate rapidly and undergo an abbreviated cell cycle, characterized by a very short G1 phase and a long S phase. Several studies revealed that the duration of $\mathrm{G} 1$ is a deter-

*Corresponding author. E-mail: hdyoun@snu.ac.kr

https://doi.org/10.5483/BMBRep.2016.49.10.161

\section{Received 22 September 2016}

Keywords: Aurkb, Cell cycle, Embryonic stem cells, Oct4 resetting, PP1

Abbreviations: Aurkb, aurora kinase b; ESCs, embryonic stem cells; Oct4, octamer binding protein 4; PP1, protein phosphatase 1

Perspective to: Shin J, Kim TW, Kim H et al, 2016, Aurkb/PP1Mediated Resetting of Oct4 During the Cell Cycle Determines the Identity of Embryonic Stem Cells, eLife;5:e10877 http://dx.doi.org/10.7554/eLife.10877 minant of maintaining pluripotency and lineage commitment. It was demonstrated that the $S$ and $G 2$ phases tend to maintain the pluripotent state at the early time of differentiation. Therefore, the cell cycle regulation in ESCs is deeply linked to maintaining ESC identity.

Even though little is known about the molecular associations between the cell cycle and pluripotency in ESCs, recent evidences implicate that core transcription factors might be responsible for the pluripotent cell cycle. It has been reported that Nanog and Sox2 are responsible for the pluripotent cell cycle. In addition, Oct4, the master regulator of ESCs, can affect the cell cycle of ESCs in both a transcriptional and non-transcriptional manner. Despite the growing evidence of a direct link between the cell cycle and pluripotency, little is known about how ESCs maintain their pluripotency through cell cycle progression and pluripotency transcriptional programs during the transition from mitosis to G1 phase. To answer this question, we focused on spatiotemporal regulation of Oct4 by the Aurora kinase b (Aurkb)-protein phosphatase 1 (PP1) axis during the cell cycle.

The Aurkb-PP1 axis is well-known for regulating the balance between phosphorylation and dephosphorylation of substrates during cell cycle progression. However, a detailed role of for the Aurkb-PP1 axis to regulate pluripotency and differentiation has not been reported. During G2/M phase of ESCs, Aurkb binds and phosphorylates the serine 229 residue of Oct4, which is a well-conserved residue throughout many species. Consistent with findings from previous reports, we found that the phosphorylation of Oct4 at serine 229 leads to its dissociation from chromatin during G2/M phase by immunostaining and a ChIP-qPCR assay. In addition, a phosphormimetic form of Oct4(S229D) rarely binds to DNA.

Most transcriptional machinery proteins, such as RNA pol II and many transcription factors dissociate from mitotic chromatin through phosphorylation. When the cell cycle resets, these transcriptional machinery proteins for ESC pluripotency should be reset. In the case of Oct4, PP1 governs the resetting during the $\mathrm{NG1}$ transition, by dephosphorylating serine 229 residues of Oct4. Through this, dephosphorylated Oct4 upregulates target genes when cells start to enter G1 phase. 
PP1 regulates the resetting of cell cycle machinery, through binding to specific sequences- the RVxF motif-and dephosphorylating interactors. We identified an RVWF motif in the C-terminal POU-h domain of Oct4 near the Aurkb phosphorylation site (S229), in the 3-dimensional structure. When we altered the phenylalanine-271 of the RVWF motif to alanine in Oct4, this mutant Oct4 bound weakly to PP1 and finally failed to reset in ESCs. Consistent with this findings, incubating ESCs with okadaic acid, a PP1 inhibitor, also impedes the reset of Oct4.

When Oct4 resets during M/G1 transition, its target genes include not only pluripotency genes but also cell cycle related genes. Especially, we found that Oct4 can govern the cell cycle of ESCs by strongly targeting two cell cycle genes, Bub1 and Rif1. Considering that Bub1 and Rif1 are important for ESCs cell cycle and pluripotency, the regulation of S-G2-M phase by Oct4 might also be crucial for ESCs identity. In addition, we found that, during $\mathrm{W} / \mathrm{G} 1$ transition, the reset of Oct4 occurs over a genome-wide region of ESCs, including pluripotency and cell cycle related genes by ChIP-seq.

To rule out the significance of recycling Oct4 through Aurkb/PP1 in ESC pluripotency, we examined it with the ZHBTc4 ESCs that are stably expressing wild-type Oct4(WT), a phosphor-mimic Oct4(S229D) mutant, and a PP1-bindingdefective Oct4(F271A) mutant. Both mutant-back up cells harbored more cells in G1 phase and fewer S-phase popula- tions, whereas Oct4(WT)-backup cells had a typical ESC cell cycle profile, indicating that the phosphorylation status of Oct4 is crucial for maintaining the pluripotency and cell cycle progression of ESCs. Furthermore, the reset of Oct4 mutants to target genes decreased and finally lost their pluripotency activity. In addition, these Oct4 mutants impeded somatic cell reprogramming.

Our study provides the bifurcating model of ESCs: a tight resetting of Oct4 on chromatin during the MG1 transition strengthens the pluripotency of ESCs. But upon differentiation, loose resetting of Oct4 during the MG1 transition triggers the cell cycle alterations and loss of pluripotency, and ultimately provides the window of opportunity to differentiation.

In this study, we have provided evidence that the cell cycle machinery cooperates with pluripotency transcriptional programs. Based on our study, we suggest that the potential of ESCs to differentiation might be derived from the small window of the MG1 transition by which the resetting of Oct4 is the central determinant to maintaining pluripotency or lineage commitment.

\section{ACKNOWLEDGEMENTS}

This work was supported by a National Research Foundation of Korea grant, funded by the Korean government (MSIP) (No. 2012R1A3A2048767 to H.-D. Y). 